\title{
Metabolizable energy, nitrogen balance, and ileal digestibility of amino acids in quality protein maize for pigs
}

\author{
Gerardo Mariscal-Landín ${ }^{1 *}$, Tércia Cesária Reis de Souza ${ }^{2}$ and Ericka Ramírez Rodríguez ${ }^{1}$
}

\begin{abstract}
Background: To compare the nutritional value and digestibility of five quality protein maize (QPM) hybrids to that of white and yellow maize, two experiments were carried out in growing pigs. In experiment 1, the energy metabolizability and the nitrogen balance of growing pigs fed one of five QPM hybrid diets were compared against those of pigs fed white or yellow maize. In experiment 2, the apparent and standardized ileal digestibility (AID and SID, respectively) of proteins and amino acids from the five QPM hybrids were compared against those obtained from pigs fed white and yellow maize. In both experiments, the comparisons were conducted using contrasts.

Results: The dry matter and nitrogen intakes were higher in the pigs fed the QPM hybrids $(P<0.05)$ than in the pigs fed white or yellow maize. Energy digestibility $(P<0.001)$ and metabolizability $(P<0.01)$ were higher in the pigs fed the white and yellow maize diets than in those fed the QPM diets. The AID of lysine was higher $(P<0.01)$ in the QPM diets than in the white and yellow maize. The AIDs of leucine, isoleucine, valine, phenylalanine, and methionine were lower in the QPM diets than those of maize (white and yellow) (all $P<0.05$ ). Maize (white and yellow) had greater SIDs of leucine, isoleucine, valine, phenylalanine, glutamic acid, serine, alanine, tyrosine, and proline $(P<0.05)$.
\end{abstract}

Conclusions: Based on these results, it was concluded that QPM had a lower metabolizable energy content and a higher amount of digestible lysine than normal maize.

Keywords: Amino acids, Energy balance, Maize, Nitrogen balance, Pigs

\section{Background}

Maize (Zea mays) is the most widely harvested cultivar in the world, and it is often used as the principal source of energy for pigs. It is also an important source of protein and amino acids in finishing pigs [1]. There are varieties of maize, other than yellow maize, that contain different nutrient concentrations [2]. Owing to these differences in nutrient density and/or composition, new types of maize such as quality protein maize (QPM) may offer nutritional advantages over the conventional yellow maize varieties.

Maize protein is deficient in the amino acids, lysine and tryptophan, which limits its value for monogastric animals [3]. Mertz et al. [4] first reported the mutant maize called Opaque 2 in 1963, which has a higher

\footnotetext{
* Correspondence: mariscal.gerardo@inifap.gob.mx

${ }^{1}$ CENID Fisiología, Instituto Nacional de Investigaciones Forestales Agrícolas y Pecuarias, km 1 Carretera a Ajuchitlán, 76280 Querétaro, México

Full list of author information is available at the end of the article
}

content of these amino acids. However, maize Opaque 2 had a soft endosperm that made it susceptible to pests and crop storage problems, for which its production was ceased. Subsequent conventional breeding efforts by the International Maize and Wheat Improvement Centre (CIMMYT) generated numerous cultivars with improved agronomic characteristics, collectively referred to as QPM [5], a type of maize with a hard endosperm rich in lysine and tryptophan due to a change in the ratio of 19- and $22-\mathrm{kD} \alpha$ zeins, an increment of $27 \mathrm{kD} \gamma$ zein [6], and a non zein protein called elongation factor $1 \alpha(\mathbf{E F}-\mathbf{1} \boldsymbol{\alpha})$ [7]. The substantial reduction in synthesis of $\alpha$-zeins results in smaller, less numerous protein bodies and a concomitant increase in non-zein endosperm proteins [3].

The metabolizable energy (ME) and amino acid (AA) digestibility of yellow maize have been extensively investigated and summarized in previous publications $[8,9]$. This allows for the accurate formulation of yellow maize-based diets for pigs to meet ME and digestible AA 
requirements. However, there is currently limited information about the nutritive value of QPM.

Therefore, the first aim of the current study was to compare the energy metabolizability and nitrogen balance of QPM to those of yellow and white maize, and the second aim was to compare the apparent and standardized ileal digestibility (AID and SID, respectively) of protein and AAs in QPM hybrids to those of yellow and white maize.

\section{Material and methods}

This study was approved by the Scientific Associate Technical Group Committee of CENID Physiology. Two experiments were conducted at the experimental farm of CENID-Physiology (INIFAP, México). The experimental animals were treated according to the guidelines of the International Guiding Principles for Biomedical Research Involving Animals [10] and the Official Mexican Standard for production, care and use of laboratory animals [11].

\section{Raw materials}

The following QPM hybrids were evaluated: 538Ta, 537Ta, 537Ig, 334Ce, and QPM1. Evaluation was also conducted on one white and one yellow maize obtained from a commercial supplier in the state of Guanajuato, Mexico. The chemical composition of these materials is shown in Table 1.

\section{Energy metabolizability and nitrogen balance (Experiment 1)}

Four consecutive groups of seven Landrace $\mathrm{x}$ Duroc pigs with a mean weight of $61.2 \pm 2.6 \mathrm{~kg}$ were used (28 pigs in total, four replicates by treatment). The pigs were placed in individual metabolic cages equipped with a self-feeder and a low-pressure drinking nipple connected to a watering system that controlled the water supply. Screens were placed under the floors, which allowed for total collection of feces and urine. The room temperature ranged from $19-22^{\circ} \mathrm{C}$.

The pigs were fed twice daily at $0800 \mathrm{~h}$ and $1800 \mathrm{~h}$. The experimental diets (Table 2) were prepared with one QPM or one maize (white or yellow) as the sole protein and energy source. The diets contained calcium carbonate, dicalcium phosphate, salt, and premixed vitamins and minerals. Chromic oxide (3 g/kg of feed) was included as an indigestible marker. The feed intake of the pigs was 2.5 times their digestible energy (DE) requirement for maintenance $\left(460 \mathrm{~kJ} / \mathrm{kg} \mathrm{BW}^{0.75}\right.$ [12]) as recommended by Adeola [13] for pigs weighing $>50 \mathrm{~kg}$. The water container in each metabolic cage was filled just before each meal to restrict water intake to $2.5 \mathrm{~L} / \mathrm{kg}$ of dry matter (DM) intake [13].

The experimental period lasted for $10 \mathrm{~d}$ for each consecutive group: $5 \mathrm{~d}$ for adaptation and $5 \mathrm{~d}$ for the total collection of feces and urine. The feces were frozen and kept at $-20^{\circ} \mathrm{C}$. At the end of the experimental period, the feces were defrosted and homogenized to obtain $10 \%$ of the weight as a final sample for lyophilizing. Urine was collected via funnels underneath the urine collection tray. This collection system included a glass wool mat to avoid contamination with feed or feces. To reduce urine $\mathrm{pH}$ and avoid $\mathrm{NH}_{3}$ volatilization, $40 \mathrm{~mL}$ of $\mathrm{HCl} 6 \mathrm{~mol} / \mathrm{L}$ were added to each urine container twice a day. The urine was removed twice a day, weighed, and filtered again through four layers of cheesecloth into a clean container. Then a $5 \%$ aliquot was taken and kept at $-20^{\circ} \mathrm{C}$ until analysis. Three urine subsamples of $20 \mathrm{~mL}$ per pig were lyophilized to measure energy [14].

\section{Ileal digestibility (Experiment 2)}

Seven Landrace $x$ Duroc pigs with a mean weight of $62.3 \pm 4.9 \mathrm{~kg}$ at the time of data collection were used. When the pigs weighed $45 \mathrm{~kg}$, a T-cannula was fitted at the terminal ileum [15]. After surgery, the pigs were placed in individual metabolism cages that included a self-feeder and a low-pressure drinking nipple. The room temperature ranged from $19-22^{\circ} \mathrm{C}$.

The post-operative period lasted for $21 \mathrm{~d}$. During this period, the pigs were fed a grower diet $(160 \mathrm{~g}$ of $\mathrm{CP} / \mathrm{kg}$ ) twice daily at $0800 \mathrm{~h}$ and $1800 \mathrm{~h}$. The amount fed was increased $100 \mathrm{~g} / \mathrm{d}$ until the level of intake was the same as that prior to surgery.

During the experimental period, the pigs received one of the experimental diets (Table 3). The diets were formulated using maize as the sole source of dietary protein. To avoid the effect of the level of the dietary protein on protein apparent digestibility [16], maize starch was used to ensure that the experimental diets consisted of the same protein level despite the different protein levels of the maize. All experimental diets contained calcium carbonate, dicalcium phosphate, and salt. To reduce dust, $20 \mathrm{~g} / \mathrm{kg}$ of maize oil was included. Vitamins and minerals exceeded the NRC [8] requirements. Chromic oxide ( $3 \mathrm{~g} / \mathrm{kg}$ of feed) was included as an indigestible marker. The feed intake of the pigs was 2.5 times their digestible energy (DE) requirement for maintenance, $460 \mathrm{~kJ} / \mathrm{kg} \mathrm{BW} \mathrm{W}^{0.75}$ [12]. The animals had free access to water.

The four experimental periods lasted seven d each: $5 \mathrm{~d}$ for adaptation and $2 \mathrm{~d}$ for digesta collection. Ileal digesta were collected continuously over a period of $10 \mathrm{~h}$ $(0800 \mathrm{~h}-1800 \mathrm{~h})$ using plastic bags $(11 \mathrm{~cm} \times 5 \mathrm{~cm})$ that contained $10 \mathrm{~mL}$ of a $0.2 \mathrm{~mol} / \mathrm{L} \mathrm{HCl}$ solution to inhibit bacterial activity and were attached to the cannula using a rubber band. When the collecting bags were full, the ileal digesta was transferred to a container and frozen at $-20^{\circ} \mathrm{C}$ until lyophilisation. 
Table 1 Chemical composition of maize $(\mathrm{g} / \mathrm{kg})^{1}$

\begin{tabular}{|c|c|c|c|c|c|c|c|}
\hline \multirow[b]{2}{*}{ Nutrient, $\mathrm{g} / \mathrm{kg}$} & \multicolumn{5}{|c|}{$\mathrm{QPM}^{2}$} & \multicolumn{2}{|c|}{ Maize } \\
\hline & QPM1 & $334 \mathrm{Ce}$ & $537 \mathrm{Ta}$ & $537 \mathrm{lg}$ & $538 \mathrm{Ta}$ & White & Yellow \\
\hline Dry Matter & 910.7 & 911.4 & 893.0 & 909.0 & 911.2 & 911.0 & 910.8 \\
\hline Protein & 85.0 & 84.0 & 86.0 & 85.0 & 87.0 & 80.0 & 75.0 \\
\hline NDF & 114.0 & 91.0 & 102.0 & 84.0 & 89.0 & 104.0 & 94.0 \\
\hline ADF & 48.0 & 39.0 & 40.0 & 38.0 & 35.0 & 34.0 & 27.0 \\
\hline Ether Extract & 34.0 & 34.0 & 47.0 & 39.0 & 52.0 & 39.0 & 37.0 \\
\hline Ash & 17.0 & 14.0 & 16.0 & 17.0 & 17.0 & 11.0 & 12.0 \\
\hline Gross Energy, MJ/kg & 16.7 & 16.6 & 16.4 & 16.0 & 16.6 & 16.5 & 16.5 \\
\hline \multicolumn{8}{|l|}{ Amino acids } \\
\hline Alanine & 5.1 & 4.9 & 4.9 & 5.0 & 4.2 & 5.9 & 5.6 \\
\hline Arginine & 5.9 & 3.5 & 5.3 & 5.3 & 4.9 & 3.8 & 3.6 \\
\hline Aspartic acid & 6.7 & 4.6 & 6.5 & 6.4 & 5.4 & 5.4 & 5.3 \\
\hline Cystine & 3.5 & 3.5 & 2.4 & 3.5 & 3.4 & 3.0 & 3.3 \\
\hline Glutamic acid & 14.1 & 12.6 & 13.6 & 14.4 & 11.4 & 15.3 & 14.2 \\
\hline Glycine & 4.1 & 2.9 & 3.9 & 4.0 & 3.5 & 3.1 & 3.0 \\
\hline Histidine & 3.5 & 2.7 & 3.4 & 2.5 & 2.6 & 2.5 & 2.1 \\
\hline Isoleucine & 2.6 & 2.0 & 2.5 & 2.4 & 2.1 & 2.6 & 2.5 \\
\hline Leucine & 7.3 & 7.7 & 7.1 & 7.3 & 5.8 & 9.6 & 9.1 \\
\hline Lysine & 3.1 & 2.7 & 2.9 & 2.7 & 2.7 & 2.0 & 2.0 \\
\hline Methionine & 1.8 & 1.8 & 2.2 & 2.3 & 2.3 & 1.5 & 2.1 \\
\hline Phenylalanine & 3.9 & 3.1 & 3.4 & 3.9 & 2.9 & 4.2 & 3.9 \\
\hline Proline & 8.4 & 7.4 & 9.4 & 8.0 & 7.9 & 9.2 & 8.9 \\
\hline Serine & 3.7 & 3.1 & 3.6 & 3.9 & 3.1 & 3.7 & 3.5 \\
\hline Threonine & 3.3 & 2.4 & 3.0 & 3.5 & 2.7 & 2.8 & 2.6 \\
\hline Tyrosine & 2.6 & 2.2 & 2.4 & 2.2 & 2.1 & 2.6 & 2.5 \\
\hline Valine & 4.7 & 3.1 & 5.4 & 4.4 & 4.6 & 3.9 & 4.3 \\
\hline
\end{tabular}

${ }^{1}$ As fed basis.

${ }^{2}$ Quality Protein Maize.

At the end of the experiment, narcosis was induced by $\mathrm{CO}_{2}$ inhalation, followed by euthanasia by exsanguination. A post-mortem examination for fistula along the length of the small intestine was performed to verify its integrity.

\section{Chemical analysis}

The raw materials, diets, digesta, and feces were ground using a laboratory mill (Arthur H. Thomas Co., Philadelphia, PA) to pass through a $0.5 \mathrm{~mm}$ mesh sieve. DM and nitrogen analysis methods, 934.01 and 976.05 [17], respectively, were performed. Chromium analysis was performed as described by Fenton and Fenton [18]. Gross energy was estimated using an adiabatic bomb calorimeter (model 1281, Parr, Moline, IL). Samples from the raw materials, diets, and digesta were hydrolyzed at $110^{\circ} \mathrm{C}$ for $24 \mathrm{~h}$ in $6 \mathrm{~mol} / \mathrm{L} \mathrm{HCl}$ to use in $\mathrm{AA}$ analysis method 994.12 [17]. For methionine and cystine analyses, oxidation with performic acid was carried out before acid hydrolysis [17]. Tryptophan was not estimated. AA analysis was performed using reverse phase HPLC (1100 HPLC Hewlett Packard), according to Henderson et al. [19]. Nitrogen in the liquid urine was estimated according to AOAC [17] method 976.05. Energy in the lyophilized urine was estimated according to Le Bellego [14].

\section{Calculations}

The AID or apparent total tract digestibility (ATTD) was estimated using the equation proposed by Fan and Sauer [20]:

$$
A I D=[1-((I D \times A F) /(A D \times I F))] \times 100
$$

where AID = apparent digestibility (ileal or total tract) of a nutrient in the diet, ID = concentration of the marker in the diet $(\mathrm{mg} / \mathrm{kg} \mathrm{DM}), \mathbf{A F}=$ concentration of the nutrient in ileal digesta or feces $(\mathrm{mg} / \mathrm{kg} \mathrm{DM})$, 
Table 2 Composition of the experimental diets (g/kg): experiment $1^{1}$

\begin{tabular}{|c|c|c|c|c|c|c|c|}
\hline \multirow[t]{2}{*}{ Ingredient, g/kg } & \multicolumn{5}{|c|}{$\mathrm{QPM}^{2}$} & \multicolumn{2}{|c|}{ Maize } \\
\hline & QPM1 & $334 \mathrm{Ce}$ & $537 \mathrm{Ta}$ & 537lg & $538 \mathrm{Ta}$ & White & Yellow \\
\hline QPM1 & 967.5 & & & & & & \\
\hline 334Ce & & 967.5 & & & & & \\
\hline $537 \mathrm{Ta}$ & & & 967.5 & & & & \\
\hline $537 \lg$ & & & & 967.5 & & & \\
\hline 538Ta & & & & & 967.5 & & \\
\hline White & & & & & & 967.5 & \\
\hline Yellow & & & & & & & 967.5 \\
\hline Calcium Phosphate & 18.5 & 18.5 & 18.5 & 18.5 & 18.5 & 18.5 & 18.5 \\
\hline Calcium Carbonate & 5.5 & 5.5 & 5.5 & 5.5 & 5.5 & 5.5 & 5.5 \\
\hline Salt & 3.5 & 3.5 & 3.5 & 3.5 & 3.5 & 3.5 & 3.5 \\
\hline Minerals $^{3}$ & 1.0 & 1.0 & 1.0 & 1.0 & 1.0 & 1.0 & 1.0 \\
\hline$V_{i t a m i n s}{ }^{4}$ & 1.0 & 1.0 & 1.0 & 1.0 & 1.0 & 1.0 & 1.0 \\
\hline Chromic oxide & 3.0 & 3.0 & 3.0 & 3.0 & 3.0 & 3.0 & 3.0 \\
\hline \multicolumn{8}{|c|}{ Chemical composition $^{5}$} \\
\hline Dry Matter & 906.6 & 903.3 & 891.1 & 904.6 & 901.4 & 905.6 & 904.8 \\
\hline Protein & 82.2 & 81.3 & 83.2 & 82.2 & 84.2 & 77.4 & 72.6 \\
\hline Gross Energy, MJ/kg & 16.5 & 16.2 & 16.1 & 15.9 & 16.1 & 16.0 & 16.0 \\
\hline
\end{tabular}

${ }^{1}$ As fed basis.

${ }^{2}$ Quality Protein Maize.

${ }^{3}$ Furnished by kg of feed: Cl 1.65 g, Na 0.87 g, Cu 7.7 mg, Fe 89.25 mg, Mn 19.98 mg, Se 0.087 mg, I 0.053 mg.

${ }^{4}$ Furnished by kg of feed: Vitamin A 6,600 IU, D 660 IU, E 100 IU, Choline 350 mg, Niacin 54 mg, Pantothenic acid 13.15 mg, Riboflavin 2.2 mg, B 1236 Mg

${ }^{5}$ Analyzed values, on an as fed basis.

$\mathbf{A D}=$ concentration of the nutrient in the diet $(\mathrm{mg} / \mathrm{kg} \mathrm{DM})$, and $\mathbf{I F}=$ concentration of the marker in the ileal digesta or feces (mg/kg DM).

The SID was estimated using the formula proposed by Furuya and Kaji [21]:

$$
S I D=[\text { AID }+(\text { Endogenous } / \text { Dietary Content })] \times 100
$$

where Endogenous = endogenous losses of a nutrient in $\mathrm{mg} / \mathrm{kg}$ DM intake, and Dietary content = amount of nutrient consumed in $\mathrm{mg} / \mathrm{kg}$ DM intake. The calculations were performed using endogenous values reported by Mariscal-Landin and Reis de Souza [22].

The ME was obtained using the formula proposed by Adeola [13]:

$$
M E=[(G E-F E-U E) / G E] \times 100
$$

where $\mathrm{ME}=$ metabolizable energy in $\mathrm{MJ} / \mathrm{d}, \mathbf{G E}=$ gross energy intake in $\mathrm{MJ} / \mathrm{d}, \mathbf{F E}=$ fecal energy output in $\mathrm{MJ} / \mathrm{d}$, and $\mathbf{U E}=$ urine energy output in $\mathrm{MJ} / \mathrm{d}$.

\section{Statistical analyses}

\section{Experiment 1}

Data were analyzed as a randomized complete block design [23] with four blocks of seven animals each and using the GLM procedure in SAS v9.2 [24]. The variables were the DM amount, nitrogen intake, energy intake, the amount of nitrogen and energy excreted in feces and urine, the apparent total tract digestibility of DM, nitrogen, and energy, nitrogen balance, and energy metabolizability. An alpha value of 0.05 was used to assess significance.

\section{Experiment 2}

Data were analyzed using a Latin square with additional columns, or a $4 \times 7$ "Youden square" [23], which included seven animals, seven treatments, and four experimental periods. The experimental variables were the protein and amino acids AID and SID of the maize. Data were analyzed using the GLM procedure in SAS v9.2 [24]. An alpha value of 0.05 was used to assess significance.

In both experiments, the means were compared using Duncan's method and the QPM (QPM1, 334Ce, 537Ta, $537 \mathrm{Ig}$, or $538 \mathrm{Ta}$ ) and normal maize (white or yellow) were compared using contrasts [23].

\section{Results}

Energy metabolizability and nitrogen balance Energy metabolizability, QPM vs normal maize

Energy intake was similar $(P>0.05)$ between the treatments: $29.5 \mathrm{MJ} / \mathrm{d}$ of QPM or normal maize. Apparent 
Table 3 Composition of the experimental diets $(\mathrm{g} / \mathrm{kg})$ : experiment $\mathbf{2}^{1}$

\begin{tabular}{|c|c|c|c|c|c|c|c|}
\hline \multirow[t]{2}{*}{ Ingredient, $\mathrm{g} / \mathrm{kg}$} & \multicolumn{5}{|c|}{$\mathrm{QPM}^{2}$} & \multicolumn{2}{|c|}{ Maize } \\
\hline & QPM1 & $334 \mathrm{Ce}$ & 537Ta & $537 \mathrm{lg}$ & $538 \mathrm{Ta}$ & White & Yellow \\
\hline QPM1 & 845.5 & & & & & & \\
\hline 334Ce & & 845.5 & & & & & \\
\hline 537Ta & & & 825.5 & & & & \\
\hline $537 \mathrm{lg}$ & & & & 835.5 & & & \\
\hline $538 \mathrm{Ta}$ & & & & & 816.5 & & \\
\hline White & & & & & & 887.5 & \\
\hline Yellow & & & & & & & 947.5 \\
\hline Maize starch & 102.0 & 102.0 & 122.0 & 112.0 & 131.5 & 60.0 & \\
\hline Calcium Phosphate & 18.5 & 18.5 & 18.5 & 18.5 & 18.5 & 18.5 & 18.5 \\
\hline Calcium Carbonate & 5.5 & 5.5 & 5.5 & 5.5 & 5.5 & 5.5 & 5.5 \\
\hline Maize oil & 20.0 & 20.0 & 20.0 & 20.0 & 20.0 & 20.0 & 20.0 \\
\hline Salt & 3.5 & 3.5 & 3.5 & 3.5 & 3.5 & 3.5 & 3.5 \\
\hline Minerals ${ }^{3}$ & 1.0 & 1.0 & 1.0 & 1.0 & 1.0 & 1.0 & 1.0 \\
\hline Vitamins ${ }^{4}$ & 1.0 & 1.0 & 1.0 & 1.0 & 1.0 & 1.0 & 1.0 \\
\hline Chromic oxide & 3.0 & 3.0 & 3.0 & 3.0 & 3.0 & 3.0 & 3.0 \\
\hline \multicolumn{8}{|c|}{ Chemical composition $^{5}$} \\
\hline Dry matter & 905.6 & 901.3 & 894.1 & 864.2 & 899.2 & 906.5 & 902.8 \\
\hline Protein & 71.1 & 70.8 & 70.6 & 71.0 & 71.3 & 70.5 & 71.6 \\
\hline Gross Energy, MJ/kg & 15.8 & 15.7 & 15.6 & 15.2 & 15.7 & 15.7 & 15.6 \\
\hline Alanine & 4.70 & 4.81 & 4.45 & 4.32 & 4.01 & 5.55 & 6.08 \\
\hline Arginine & 5.36 & 3.47 & 4.86 & 4.57 & 4.70 & 3.59 & 3.93 \\
\hline Aspartic acid & 6.10 & 4.54 & 5.83 & 5.59 & 5.04 & 5.04 & 5.69 \\
\hline Cystine & 3.22 & 3.47 & 2.19 & 3.13 & 3.25 & 2.82 & 3.53 \\
\hline Glutamic acid & 12.87 & 12.47 & 12.38 & 12.62 & 10.85 & 14.43 & 15.41 \\
\hline Glycine & 3.71 & 2.85 & 3.56 & 3.56 & 3.33 & 2.90 & 3.24 \\
\hline Histidine & 3.22 & 2.67 & 3.08 & 2.54 & 2.48 & 2.39 & 2.36 \\
\hline Isoleucine & 2.39 & 1.96 & 2.19 & 1.61 & 1.96 & 2.39 & 2.65 \\
\hline Leucine & 6.60 & 7.57 & 6.39 & 6.10 & 5.55 & 9.05 & 9.81 \\
\hline Lysine & 2.80 & 2.67 & 2.59 & 2.54 & 2.48 & 1.88 & 2.16 \\
\hline Methionine & 1.65 & 1.16 & 2.02 & 1.10 & 1.45 & 1.45 & 2.26 \\
\hline Phenylalanine & 3.55 & 3.12 & 3.08 & 2.80 & 2.82 & 3.93 & 4.22 \\
\hline Proline & 6.35 & 6.14 & 8.50 & 4.91 & 7.52 & 8.71 & 9.62 \\
\hline Serine & 3.38 & 3.03 & 3.24 & 3.47 & 2.90 & 3.50 & 3.73 \\
\hline Threonine & 2.97 & 2.40 & 2.75 & 3.39 & 2.56 & 2.65 & 2.85 \\
\hline Tyrosine & 2.39 & 2.23 & 2.19 & 2.12 & 1.96 & 2.48 & 2.65 \\
\hline Valine & 4.29 & 3.03 & 4.86 & 2.71 & 4.36 & 3.59 & 4.71 \\
\hline
\end{tabular}

${ }^{1}$ As fed basis.

${ }^{2}$ Quality Protein Maize.

${ }^{3}$ Furnished by kg of feed: Cl 1.65 g, Na 0.87 g, Cu 7.7 mg, Fe 89.25 mg, Mn 19.98 mg, Se 0.087 mg, I 0.053 mg.

${ }^{4}$ Furnished by kg of feed: Vitamin A 6,600 IU, D 660 IU, E 100 IU, Choline 350 mg, Niacin 54 mg, Pantothenic acid 13.15 mg, Riboflavin 2.2 mg, B 1236 Mg.

${ }^{5}$ Analyzed values, on an as fed basis.

total tract digestibility was lower in the pigs fed QPM (89.8) than in those fed normal maize (91.0, $P<0.001)$. While UE was similar $(0.58 \mathrm{MJ} / \mathrm{d}, P>0.05)$ between all the treatments, the metabolizability of energy was lower in the pigs fed QPM (87.9) than in those fed normal maize (88.9) (Table 4). 
Table 4 Energy metabolizability and nitrogen balance: experiment $1^{1}$

\begin{tabular}{|c|c|c|c|c|c|c|c|c|c|c|c|}
\hline \multirow[t]{2}{*}{ Traits } & \multicolumn{5}{|c|}{$\mathrm{QPM}^{2}$} & \multicolumn{2}{|c|}{ Maize } & \multirow[b]{2}{*}{$\mathrm{SEM}^{3}$} & \multicolumn{2}{|c|}{ Contrast } & \multirow[b]{2}{*}{ Prob $^{4}$} \\
\hline & QPM1 & $334 C e$ & $537 \mathrm{Ta}$ & $537 \mathrm{lg}$ & $538 \mathrm{Ta}$ & White & Yellow & & QPM & Normal & \\
\hline \multicolumn{12}{|l|}{ Dry Matter, kg/d } \\
\hline Intake & $1.67^{\mathrm{a}}$ & $1.66^{\mathrm{a}}$ & $1.66^{\mathrm{a}}$ & $1.68^{\mathrm{a}}$ & $1.64^{\mathrm{a}}$ & $1.66^{\mathrm{a}}$ & $1.55^{\mathrm{b}}$ & 0.010 & 1.66 & 1.60 & 0.05 \\
\hline \multicolumn{12}{|l|}{ Energy, $\mathrm{MJ} / \mathrm{d}$} \\
\hline Intake & $29,9^{\mathrm{a}}$ & $29.7^{\mathrm{a}}$ & $29.7^{\mathrm{a}}$ & $30.2^{\mathrm{a}}$ & $29.5^{\mathrm{a}}$ & $29.7^{\mathrm{a}}$ & $27.7^{b}$ & 0.17 & 29.8 & 28.7 & 0.63 \\
\hline In feces & $3.1^{\mathrm{a}}$ & $3.2^{\mathrm{a}}$ & $3.0^{\mathrm{a}}$ & $2.9^{\mathrm{ab}}$ & $2.9^{\mathrm{ab}}$ & $2.6^{b}$ & $2.6^{\mathrm{b}}$ & 0.04 & 3.0 & 2.6 & 0.001 \\
\hline Digestible & $26.8^{\mathrm{a}}$ & $26.5^{\mathrm{a}}$ & $26.7^{\mathrm{a}}$ & $27.3^{\mathrm{a}}$ & $26.5^{\mathrm{a}}$ & $27.1^{\mathrm{a}}$ & $25.1^{\mathrm{b}}$ & 0.16 & 26.7 & 26.1 & 0.01 \\
\hline In Urine & 0.50 & 0.58 & 0.53 & 0.60 & 0.62 & 0.60 & 0.61 & 0.013 & 0.57 & 0.60 & 0.21 \\
\hline Metabolizable & $26.3^{\mathrm{a}}$ & $25.9^{\mathrm{a}}$ & $26.2^{\mathrm{a}}$ & $26.7^{\mathrm{a}}$ & $25.9^{\mathrm{a}}$ & $26.5^{\mathrm{a}}$ & $24.5^{\mathrm{b}}$ & 0.16 & 26.2 & 25.5 & 0.01 \\
\hline \multicolumn{12}{|l|}{ Nitrogen, g/d } \\
\hline Intake & $22.4^{b}$ & $24.6^{\mathrm{a}}$ & $24.5^{\mathrm{a}}$ & $24.6^{a}$ & $24.4^{\mathrm{a}}$ & $22.0^{\mathrm{b}}$ & $22.1^{b}$ & 0.13 & 24.0 & 22.0 & 0.001 \\
\hline In feces & 3.6 & 4.1 & 3.8 & 3.7 & 3.8 & 3.4 & 3.5 & 0.08 & 3.82 & 3.46 & 0.32 \\
\hline Digestible & $18.7^{b}$ & $20.5^{\mathrm{a}}$ & $20.6^{\mathrm{a}}$ & $20.4^{\mathrm{a}}$ & $20.5^{\mathrm{a}}$ & $18.5^{\mathrm{b}}$ & $18.6^{\mathrm{b}}$ & 0.35 & 20.1 & 18.6 & 0.001 \\
\hline In Urine & $10.2^{\mathrm{a}}$ & $12.6^{\mathrm{b}}$ & $13.2^{\mathrm{b}}$ & $11.9^{\mathrm{b}}$ & $12.0^{\mathrm{b}}$ & $12.1^{\mathrm{b}}$ & $12.2^{\mathrm{b}}$ & 0.21 & 11.97 & 12.2 & 0.74 \\
\hline Retained & $8.5^{\mathrm{a}}$ & $7.9^{\mathrm{ab}}$ & $7.5^{\mathrm{ab}}$ & $8.5^{\mathrm{a}}$ & $8.5^{\mathrm{a}}$ & $6.5^{\mathrm{ab}}$ & $6.4^{\mathrm{b}}$ & 0.24 & 8.18 & 6.44 & 0.19 \\
\hline \multicolumn{12}{|l|}{ Dry matter, MJ/kg } \\
\hline Digestible energy & $16.11^{\mathrm{cd}}$ & $15.96^{\mathrm{d}}$ & $16.12^{\mathrm{bcd}}$ & $16.29^{a b}$ & $16.18^{\mathrm{abc}}$ & $16.34^{\mathrm{a}}$ & $16.22^{\mathrm{abc}}$ & 0.007 & 16.13 & 16.28 & 0.01 \\
\hline Metabolizable energy & $15.59^{\mathrm{ab}}$ & $15.38^{b}$ & $15.59^{\mathrm{ab}}$ & $15.70^{\mathrm{a}}$ & $15.55^{\mathrm{ab}}$ & $15.74^{\mathrm{a}}$ & $15.61^{\mathrm{ab}}$ & 0.009 & 15.56 & 15.68 & 0.09 \\
\hline \multicolumn{12}{|l|}{ Coefficient } \\
\hline DM Digestibility & $88.7^{c}$ & $88.2^{c}$ & $88.9^{c}$ & $90.1^{\mathrm{ab}}$ & $89.2^{b c}$ & $90.8^{a}$ & $90.2^{\mathrm{a}}$ & 0.12 & 89.0 & 90.5 & 0.001 \\
\hline E Digestibility & $89.6^{\mathrm{bc}}$ & $89.1^{c}$ & $89.8^{\mathrm{bc}}$ & $90.4^{\mathrm{ab}}$ & $90.1^{b c}$ & $91.3^{\mathrm{a}}$ & $90.6^{\mathrm{ab}}$ & 0.14 & 89.8 & 91.0 & 0.001 \\
\hline Metabolizability & $87.9^{\mathrm{b}}$ & $87.2^{\mathrm{b}}$ & $88.0^{b}$ & $88.4^{\mathrm{ab}}$ & $88.0^{\mathrm{b}}$ & $89.3^{\mathrm{a}}$ & $88.4^{\mathrm{ab}}$ & 0.15 & 87.9 & 88.9 & 0.01 \\
\hline N Digestibility & 83.7 & 83.3 & 84.3 & 84.7 & 84.3 & 84.3 & 84.2 & 0.30 & 84.1 & 84.3 & 0.72 \\
\hline \multicolumn{12}{|c|}{$\mathrm{N}$ retention as percentage of } \\
\hline Intake ${ }^{5}$ & $38.2^{\mathrm{a}}$ & $32.1^{\mathrm{ab}}$ & $30.5^{\mathrm{ab}}$ & $35.2^{\mathrm{ab}}$ & $35.0^{\mathrm{ab}}$ & $29.6^{\mathrm{ab}}$ & $28.8^{\mathrm{b}}$ & 1.01 & 34.2 & 29.2 & 0.28 \\
\hline Absorbed $^{6}$ & $45.7^{\mathrm{a}}$ & $38.5^{\mathrm{ab}}$ & $36.1^{\mathrm{ab}}$ & $41.6^{\mathrm{ab}}$ & $41.3^{\mathrm{ab}}$ & $35.0^{\mathrm{b}}$ & $34.2^{\mathrm{b}}$ & 1.14 & 40.3 & 34.6 & 0.20 \\
\hline
\end{tabular}

${ }^{1}$ All data are reported on a DM basis.

${ }^{2}$ Quality Protein Maize.

${ }^{3}$ Standard error of the mean.

${ }^{4}$ Contrast Probability.

${ }^{5}(\mathrm{~N} \text { retained/ } \mathrm{N} \text { intake })^{*} 100$

${ }^{6}(\mathrm{~N}$ retained/ $\mathrm{N}$ absorbed $) * 100$.

${ }^{a b c}$ different letters in the same line differ $(P<0.05)$.

\section{Energy metabolizability, means comparison}

Energy intake was lower in the pigs fed yellow maize $(27.7 \mathrm{MJ} / \mathrm{d})$ than in the pigs fed white maize $(29.8 \mathrm{MJ} / \mathrm{d}$, $P<0.05)$. Apparent total tract digestibility was higher in the pigs fed white maize (91.3) and lower in the pigs fed 334Ce QPM (89.1, P<0.05). The metabolizability of energy was higher in the pigs fed white maize (89.3) than in the pigs fed QPM1 (87.9), except for those fed QPM 537Ig (88.4; Table 4).

\section{Nitrogen balance, QPM vs normal maize}

The DM intake was higher in the pigs fed QPM, compared to those fed normal maize (1.66 vs $1.60 \mathrm{~kg} / \mathrm{d}, P<0.05$ ), and the DM total tract digestibility was lower in the QPM pigs, compared to those fed normal maize (89.0 vs 90.5 ,
$P<0.001)$. The daily nitrogen intake was higher in the pigs fed QPM $(24.0 \mathrm{~g} / \mathrm{d})$ than in the pigs fed normal maize $(22.0 \mathrm{~g} / \mathrm{d}, P<0.001)$; however, nitrogen digestibility was similar between the treatments $(84.1, P>0.05)$. Consequently, the digestible nitrogen intake was higher in the pigs fed QPM. Urinary nitrogen excretion was similar between treatments $(12.0 \mathrm{~g} / \mathrm{d}, P>0.05)$. Nitrogen retention as a proportion of nitrogen intake or nitrogen absorption was also similar $(P>0.05)$ between the treatments (Table 4$)$.

\section{Nitrogen balance, means comparison}

Although the nitrogen intake in the pigs fed QPM1 was lower $(22.4 \mathrm{~g} / \mathrm{d})$ than in the pigs fed the other hybrid QPMs $(24.5 \mathrm{~g} / \mathrm{d}, P<0.001)$ and similar to the pigs fed normal maize $(22.0 \mathrm{~g} / \mathrm{d})$, the pigs fed QPM1 retained 
more nitrogen as a percentage of nitrogen intake (38.2\%) or nitrogen absorption (45.7\%) than the pigs fed yellow maize $(28.8 \%$ and $34.2 \%$, respectively, $P<0.05$; Table 4$)$.

\section{Ileal digestibility}

\section{Apparent ileal digestibility, QPM vs normal maize}

The DM digestibility was lower in the pigs fed QPM (78.7) than in those fed normal maize $(80.0, P<0.05)$. The $\mathrm{CP}$ digestibility was similar in all of the diets (mean 73.0, $P>0.05)$.

Lysine digestibility was higher in the pigs fed QPM than in the pigs fed normal maize $(P<0.05$; Table 5$)$. The digestibility of leucine, isoleucine, phenylalanine, glutamic acid, alanine, tyrosine, proline, valine, serine, and methionine was lower in the QPM pigs than in those fed normal maize (all $P<0.05$; Table 5).

\section{Apparent ileal digestibility, means comparison}

In general, QPM 537Ig had the lowest digestibility, except for that for arginine, threonine, serine, and glycine; for these AAs, QPM 334Ce had the lowest digestibility (Table 5). The white maize diet demonstrated lower AID of the sulphur AAs than the yellow maize diet. The digestibility of leucine, alanine, and tyrosine was lower in all of the QPM diets than in the normal maize diets (Table 5).

Standardized ileal digestibility, QPM vs normal maize The digestibility of CP, lysine, arginine, histidine, methionine, threonine, aspartic acid, glycine, and cystine was similar between QPM and normal maize (all $P>0.05$; Table 6). The digestibility of glutamic acid, tyrosine, leucine, isoleucine, phenylalanine, alanine, valine, serine, and proline was lower in the QPM diets than in the normal maize diets (all $P<0.05$; Table 6).

\section{Standardized ileal digestibility, means comparison}

The SID of QPM 537Ig was consistently the lowest among the QPMs. The digestibility of glutamic acid and tyrosine was lower in the QPM diets than in the normal maize diets (Table 6). Methionine was less digestible in the white maize diet than in the yellow maize diet (Table 6).

\section{Discussion}

Energy metabolizability and nitrogen balance

The total tract digestibility of energy in the QPM diets was $1.3 \%$ lower, on average, than the normal maize diets.

Table 5 Apparent ileal digestibility of the maize: experiment 2

\begin{tabular}{|c|c|c|c|c|c|c|c|c|c|c|c|}
\hline \multirow[t]{2}{*}{ Traits } & \multicolumn{5}{|c|}{$\mathrm{QPM}^{1}$} & \multicolumn{2}{|c|}{ Maize } & \multirow[b]{2}{*}{ SEM $^{2}$} & \multicolumn{2}{|c|}{ Contrast } & \multirow[b]{2}{*}{ Prob $^{3}$} \\
\hline & QPM1 & $334 C e$ & 537Тa & $537 \mathrm{lg}$ & $538 \mathrm{Ta}$ & White & Yellow & & QPM & Normal & \\
\hline Dry Matter & $77.5^{c}$ & $77.6^{c}$ & $81.9^{a}$ & $77.7^{c}$ & $78.6^{b c}$ & $80.9^{a b}$ & $79.1^{\mathrm{abc}}$ & 0.33 & 78.7 & 80.0 & 0.05 \\
\hline Protein & 72.0 & 74.3 & 73.4 & 71.4 & 74.3 & 71.8 & 74.1 & 0.32 & 73.1 & 72.9 & 0.77 \\
\hline \multicolumn{12}{|l|}{ Amino acids } \\
\hline Alanine & $73.2^{b}$ & $72.7^{b}$ & $71.6^{\mathrm{b}}$ & $69.0^{b}$ & $70.6^{b}$ & $79.8^{a}$ & $80.1^{a}$ & 0.67 & 71.4 & 79.9 & 0.001 \\
\hline Arginine & $82.1^{\mathrm{a}}$ & $73.8^{b}$ & $82.3^{a}$ & $81.3^{a}$ & $85.5^{a}$ & $80.0^{\mathrm{a}}$ & $82.7^{a}$ & 0.72 & 81.0 & 81.4 & 0.79 \\
\hline Aspartic acid & 75.2 & 68.4 & 75.9 & 71.5 & 71.9 & 75.4 & 75.6 & 0.61 & 72.6 & 75.5 & 0.06 \\
\hline Cystine & $72.9^{c}$ & $78.9^{\mathrm{ab}}$ & $66.9^{d}$ & $74.9^{b c}$ & $78.0^{\mathrm{abc}}$ & $75.1^{\mathrm{bc}}$ & $80.9^{a}$ & 0.56 & 74.3 & 78.0 & 0.07 \\
\hline Glutamic acid & $83.3^{b}$ & $83.1^{b}$ & $83.1^{b}$ & $81.5^{b}$ & $82.1^{b}$ & $86.9^{a}$ & $86.9^{a}$ & 0.29 & 82.6 & 86.9 & 0.001 \\
\hline Glycine & $55.1^{\mathrm{ab}}$ & $49.8^{b}$ & $58.9^{\mathrm{a}}$ & $54.6^{\mathrm{ab}}$ & $51.9^{\mathrm{ab}}$ & $55.8^{\mathrm{ab}}$ & $57.0^{\mathrm{ab}}$ & 0.91 & 54.0 & 56.4 & 0.83 \\
\hline Histidine & $87.2^{a b}$ & $83.8^{b c}$ & $88.3^{a}$ & $82.6^{c}$ & $85.4^{\mathrm{abc}}$ & $86.1^{\mathrm{abc}}$ & $84.8^{a b c}$ & 0.45 & 85.4 & 85.4 & 0.93 \\
\hline Isoleucine & $75.4^{\mathrm{ab}}$ & $69.9^{b}$ & $74.3^{\mathrm{ab}}$ & $60.2^{c}$ & $71.8^{b}$ & $79.4^{a}$ & $79.2^{a}$ & 0.66 & 70.3 & 79.3 & 0.001 \\
\hline Leucine & $82.4^{b}$ & $83.2^{b}$ & $83.1^{b}$ & $80.5^{b}$ & $82.1^{b}$ & $89.0^{a}$ & $89.4^{a}$ & 0.43 & 82.3 & 89.2 & 0.001 \\
\hline Lysine & $77.2^{\mathrm{a}}$ & $76.7^{a}$ & $77.1^{a}$ & $73.8^{\mathrm{ab}}$ & $75.1^{\mathrm{ab}}$ & $69.0^{\mathrm{b}}$ & $72.4^{\mathrm{ab}}$ & 0.72 & 76.0 & 70.7 & 0.01 \\
\hline Methionine & $80.5^{b}$ & $74.2^{c}$ & $85.5^{\mathrm{a}}$ & $70.7^{d}$ & $75.5^{c}$ & $76.8^{c}$ & $84.3^{a}$ & 0.36 & 77.3 & 80.5 & 0.05 \\
\hline Phenylalanine & $82.7^{b}$ & $80.6^{b c}$ & $81.9^{b}$ & $77.3^{c}$ & $82.6^{b}$ & $88.0^{\mathrm{a}}$ & $87.6^{a}$ & 0.46 & 81.0 & 87.7 & 0.001 \\
\hline Proline & $51.2^{\mathrm{cd}}$ & $61.6^{b c}$ & $72.5^{\mathrm{ab}}$ & $45.8^{d}$ & $61.5^{b c}$ & $71.6^{a b}$ & $80.3^{a}$ & 1.63 & 58.5 & 75.9 & 0.001 \\
\hline Serine & $71.0^{\mathrm{ab}}$ & $67.8^{b}$ & $72.0^{\mathrm{ab}}$ & $70.4^{a b}$ & $69.4^{b}$ & $76.8^{a}$ & $76.6^{a}$ & 0.70 & 70.1 & 76.6 & 0.01 \\
\hline Threonine & $60.4^{a b}$ & $54.6^{b}$ & $61.6^{\mathrm{ab}}$ & $64.1^{\mathrm{a}}$ & $60.1^{b}$ & $64.8^{a}$ & $62.4^{\mathrm{ab}}$ & 0.96 & 60.1 & 63.6 & 0.39 \\
\hline Tyrosine & $77.9^{b}$ & $76.8^{b}$ & $77.7^{b}$ & $73.8^{b}$ & $74.7^{\mathrm{b}}$ & $83.7^{\mathrm{a}}$ & $83.9^{a}$ & 0.57 & 76.2 & 83.8 & 0.001 \\
\hline Valine & $77.6^{\mathrm{a}}$ & $70.4^{b}$ & $81.7^{\mathrm{a}}$ & $63.3^{c}$ & $80.9^{a}$ & $78.5^{\mathrm{a}}$ & $82.8^{a}$ & 0.18 & 74.8 & 80.6 & 0.01 \\
\hline
\end{tabular}

${ }^{1}$ Quality Protein Maize

${ }^{2}$ Standard error of the mean.

${ }^{3}$ Probability of contrast.

abcd different letters in the same line differ $(P<0.05)$. 
Table 6 Standardized ileal digestibility of the maize: experiment 2

\begin{tabular}{|c|c|c|c|c|c|c|c|c|c|c|c|}
\hline \multirow[t]{2}{*}{ Traits } & \multicolumn{5}{|c|}{$\mathrm{QPM}^{1}$} & \multicolumn{2}{|c|}{ Maize } & \multirow[b]{2}{*}{ SEM $^{2}$} & \multicolumn{2}{|c|}{ Contrast } & \multirow[b]{2}{*}{ Prob $^{3}$} \\
\hline & QPM1 & $334 \mathrm{Ce}$ & 537Ta & 537lg & $538 \mathrm{Ta}$ & White & Yellow & & QPM & Normal & \\
\hline Protein & 86.7 & 88.0 & 88.2 & 85.7 & 88.2 & 86.9 & 88.1 & 0.32 & 87.4 & 87.5 & 0.98 \\
\hline \multicolumn{12}{|l|}{ Amino acids } \\
\hline Alanine & $80.7^{\mathrm{ab}}$ & $80.0^{b c}$ & $79.4^{c}$ & $77.1^{c}$ & $79.4^{c}$ & $86.1^{a}$ & $85.8^{\mathrm{ab}}$ & 0.66 & 79.3 & 86.0 & 0.01 \\
\hline Arginine & $87.9^{\mathrm{ab}}$ & $82.8^{b}$ & $88.8^{\mathrm{ab}}$ & $88.2^{\mathrm{ab}}$ & $92.2^{\mathrm{a}}$ & $88.8^{\mathrm{ab}}$ & $90.8^{a}$ & 0.72 & 88.0 & 89.8 & 0.65 \\
\hline Aspartic acid & 85.2 & 81.9 & 86.4 & 82.4 & 84.0 & 87.5 & 86.4 & 0.61 & 84.0 & 86.9 & 0.06 \\
\hline Cystine & $77.1^{c d}$ & $82.8^{\mathrm{ab}}$ & $73.1^{c}$ & $79.2^{\mathrm{bc}}$ & $82.1^{a b c}$ & $79.9^{\mathrm{abc}}$ & $88.7^{\mathrm{a}}$ & 0.56 & 78.9 & 82.3 & 0.10 \\
\hline Glutamic acid & $88.7^{\mathrm{b}}$ & $88.7^{\mathrm{b}}$ & $88.7^{\mathrm{b}}$ & $87.1^{\mathrm{b}}$ & $88.6^{\mathrm{b}}$ & $91.7^{\mathrm{a}}$ & $91.4 a$ & 0.28 & 88.3 & 91.6 & 0.001 \\
\hline Glycine & 73.8 & 74.2 & 78.3 & 74.2 & 72.7 & 79.7 & 78.4 & 0.91 & 74.6 & 79.0 & 0.26 \\
\hline Histidine & $92.0^{\mathrm{ab}}$ & $89.5^{\mathrm{ab}}$ & $93.3^{\mathrm{a}}$ & $88.6^{\mathrm{b}}$ & $91.5^{\mathrm{ab}}$ & $92.5^{\mathrm{ab}}$ & $91.3^{\mathrm{ab}}$ & 0.46 & 91.0 & 91.9 & 0.39 \\
\hline Isoleucine & $86.3^{\mathrm{ab}}$ & $83.3^{b}$ & $86.3^{\mathrm{ab}}$ & $76.4^{c}$ & $85.0^{\mathrm{ab}}$ & $90.3^{a}$ & $89.0^{\mathrm{ab}}$ & 0.66 & 83.4 & 89.7 & 0.01 \\
\hline Leucine & $89.2^{\mathrm{bc}}$ & $89.1^{b c}$ & $90.2^{\text {abc }}$ & $87.9^{c}$ & $90.2^{\mathrm{abc}}$ & $94.0^{a}$ & $94.0^{\mathrm{a}}$ & 0.44 & 89.3 & 94.0 & 0.01 \\
\hline Lysine & 88.1 & 88.2 & 88.9 & 85.9 & 87.5 & 85.3 & 86.6 & 0.72 & 87.7 & 85.9 & 0.18 \\
\hline Methionine & $84.4^{\mathrm{b}}$ & $79.7^{b}$ & $88.7^{\mathrm{a}}$ & $76.4^{d}$ & $79.8^{c}$ & $81.1^{c}$ & $87.1^{\mathrm{ab}}$ & 0.36 & 81.8 & 84.1 & 0.30 \\
\hline Phenylalanine & $89.5^{\mathrm{bcd}}$ & $88.4^{\complement d}$ & $89.8^{\mathrm{bcd}}$ & $86.0^{d}$ & $91.3^{\mathrm{abc}}$ & $94.2^{\mathrm{a}}$ & $93.3^{\mathrm{ab}}$ & 0.46 & 89.0 & 93.7 & 0.01 \\
\hline Proline & $70.2^{c}$ & $81.2^{a b c}$ & $86.7^{\mathrm{ab}}$ & $70.3^{c}$ & $77.5^{\mathrm{bc}}$ & $85.4^{\mathrm{ab}}$ & $92.8^{\mathrm{a}}$ & 1.63 & 77.2 & 89.1 & 0.02 \\
\hline Serine & $84.6^{\mathrm{ab}}$ & $82.9^{b}$ & $86.2^{\mathrm{ab}}$ & $83.6^{\mathrm{ab}}$ & $85.2^{\mathrm{ab}}$ & $89.9^{a}$ & $88.9^{\mathrm{ab}}$ & 0.70 & 84.5 & 89.4 & 0.03 \\
\hline Threonine & 77.7 & 76.0 & 80.2 & 79.2 & 80.2 & 84.1 & 80.4 & 0.95 & 78.6 & 82.3 & 0.35 \\
\hline Tyrosine & $83.2^{\mathrm{b}}$ & $82.5^{b}$ & $83.4^{b}$ & $79.8^{\mathrm{b}}$ & $81.1^{\mathrm{b}}$ & $88.8^{\mathrm{a}}$ & $88.7^{a}$ & 0.57 & 82.0 & 89.7 & 0.001 \\
\hline Valine & $86.0^{\mathrm{ab}}$ & $82.3^{b}$ & $89.1^{a}$ & $76.6^{c}$ & $89.1^{a}$ & $88.5^{\mathrm{a}}$ & $90.4^{a}$ & 0.58 & 84.6 & 89.5 & 0.02 \\
\hline
\end{tabular}

${ }^{1}$ Quality Protein Maize.

${ }^{2}$ Standard error of the mean.

${ }^{3}$ Probability.

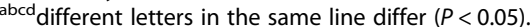

While urinary energy was similar between the diets, metabolizability was also lower in the QPM diets than in the white maize diet.

The starch type of QPM may explain its lower digestibility in the current study; amylose is negatively correlated with average daily gain [25]. Furthermore, the starch of waxy sorghums, which are low in amylose, is more digestible than the starch of non-waxy sorghums [26]; this has also been demonstrated with maize starch in ducks [27]. Although QPM has a vitreous endosperm phenotype, it is rich in a no-crystalline amylopectin that forms bonds with $\gamma$ zein (27-kDa) [28].

The negative effect of fiber on energy digestibility $[29,30]$ could provide another feasible explanation; dietary fiber is less digestible than other nutrients such as starch, sugars, fat, or protein $(<50 \%$ vs $80-100 \%)$ [31]. Moreover, corn fiber is essentially insoluble [32]; QPM had a higher ADF content than the normal maize (40 g/ $\mathrm{kg}$ QPM vs $30 \mathrm{~g} / \mathrm{kg}$ maize), and this could have resulted in considerable effects on digestibility given that maize fiber, in general, is poorly digested by growing pigs [31]. Moreover, the ME:DE ratio in the current study was 0.98 , which is similar to the 0.96 estimated by Noblet and van Milgen [31].
Generally, $50 \%$ of the nitrogen that is absorbed is retained in the body, and the other $50 \%$ is excreted in urine [29]. The retention of nitrogen in the current study was lower (37.5\%), which could be attributed to a diet of low protein quality. Maize protein is deficient in lysine and tryptophan [3], and it is well-known that nitrogen retention in growing pigs is related to the lysine level in the diet as lysine is the first limiting AA [33]. Lysine digestibility was higher in the pigs fed QPM1 maize, and these same pigs retained $26 \%$ more nitrogen $(1.7 \mathrm{~g} / \mathrm{d})$. These results are consistent with previous reports based on animal studies $[1,34,35]$, as well as in humans where the consumption of QPM by children resulted in a $12 \%$ increase in weight [34] and a $9 \%$ increase in height and weight [36].

\section{Ileal digestibility}

The average AA profile of the proteins in the QPM was different from that of yellow and white maize. QPM had more lysine (45\%), arginine (37\%), histidine (31\%), glycine (23\%), methionine (19\%), threonine (13\%), aspartic acid (13\%), valine (10\%), and cystine (7\%) than white and yellow maize. In contrast, QPM had less leucine (-23\%), alanine $(-14 \%)$, phenylalanine $(-13 \%)$, glutamic acid $(-8 \%)$, 
tyrosine $(-8 \%)$, and proline $(-7 \%)$ than white and yellow maize. Other studies have also reported that QPM is rich in lysine $[5,37,38]$, while the low leucine and proline content is associated with a decrease in zein protein [6].

Dietary protein content affects apparent digestibility $[16,39,40]$. To avoid this effect, the experimental diets were iso-nitrogenous, resulting in similar protein digestibility in all of the diets. However, the differences in amino acid content may explain the differences in digestibility. It has been previously reported that the high lysine content in QPM results in a higher AID $[34,35]$, as was found in the current study. Similarly, the high leucine, phenylalanine, glutamic acid, alanine, tyrosine, and proline content of normal maize (white and yellow) could explain the high AID that was observed. Moreover, the low AID observed for threonine may be caused by its richness within the endogenous protein $[22,41,42]$.

Although the use of the estimate from one endogenous protein in each experiment has been recommended to estimate the SID $[39,40]$, it is also true that the SID has been estimated from previously published AID data and corrected with an endogenous protein that was estimated later $[9,43,44]$ or previously $[45,46]$. This supports the use of a "standard" endogenous protein to correct the AID. The SID removes the effect of nutrient level on the digestibility value by correcting for basal endogenous losses $[39,43,47]$. The SID of lysine was similar in all of the maize diets. Additionally, when SID was estimated, threonine reached similar values to those of the other amino acids; this may be related to its richness within the endogenous protein. The SID coefficients estimated for maize in the present work were similar to those reported in previous studies $[8,9,43]$. However, no values for SID have been reported previously for QPM.

\section{Conclusions}

The energy furnished by QPM was used less efficiently (metabolizability) than the energy furnished by normal maize. The AID of lysine was higher in the QPM than in the normal maize; however, the SID of lysine was similar between QPM and normal maize. The current study provides additional data about the nutrient composition, AA digestibility, and nitrogen utilization of QPM.

\footnotetext{
Abbreviations

CIMMYT: International maize and wheat improvement centre; QPM: Quality protein maize; EF-1 a: Elongation factor 1a; DM: Dry matter; DE: Digestible energy; ME: Metabolizable energy; AA: Amino acid; ADF: Acid detergent fiber; AID: Apparent ileal digestibility; SID: Standardized ileal digestibility; ATTD: Apparent total tract digestibility.
}

\section{Competing interests}

The authors declare that they have no competing interests.

\section{Authors' contributions}

GML conceived and designed the study; ERR carried out the lab analysis; TCRS contributed to data analysis; GML and TCRS wrote the manuscript. All authors read and approved the final manuscript.

\section{Acknowledgements}

To National Research Institute for Forestry, Crops and Livestock for the financial support given to this research project.

\section{Author details}

${ }^{1}$ CENID Fisiología, Instituto Nacional de Investigaciones Forestales Agrícolas y Pecuarias, km 1 Carretera a Ajuchitlán, 76280 Querétaro, México. ${ }^{2}$ Facultad de Ciencias Naturales, Universidad Autónoma de Querétaro, México, Av. de las Ciencias s/n, 76230 Querétaro, México.

Received: 8 January 2014 Accepted: 22 April 2014

Published: 7 May 2014

\section{References}

1. Cantarelli VS, Fialho ET, de Sousa RV, de Freitas RTF, Lima JAF: Composição química, vitreosidade e digestibilidade de diferentes híbridos de milho para suínos. Ciênc Agrotec 2007, 31:860-864.

2. Snow JL, Stein HH, Ku PK, Trottier NL: Amino acid digestibility and nitrogen utilization of high oil, high lysine, and waxy maize fed to growing pigs. Anim Feed Sci Technol 2004, 113:113-126.

3. Jia M, Wu H, Clay K, Jung R, Larkins B, Gibbon B: Identification and characterization of lysine-rich proteins and starch biosynthesis genes in the opaque2 mutant by transcriptional and proteomic analysis. BMC Plant Biology 2013, 13:60.

4. Mertz ET, Bates LS, Nelson OE: Mutant gene that changes protein composition and increases lysine content of maize endosperm. Science 1964, 145:279-280.

5. Nuss ET, Tanumihardjo SA: Quality protein maize for Africa: closing the protein inadequacy gap in vulnerable populations. Adv Nutr 2011, 2:217-224.

6. Wallace JC, Lopes MA, Paiva E, Larkins BA: New methods for extraction and quantitation of zeins reveal a high content of $\gamma$-zein in modified opaque-2 maize. Plant Physiol 1990, 92:191-196.

7. Habben JE, Moro GL, Hunter BG, Hamaker BR, Larkins BA: Elongation factor 1a concentration is highly correlated with the lysine content of maize endosperm. PNAS 1995, 92:8640-8644.

8. NRC: Nutrient requirements of swine. 10th edition. Washington, DC: The National Academy Press; 1998.

9. INRA: Tables de composition et de valeur nutritive des matières premières destinées aux animaux d'élevage. Porcs, volailles, bovins, ovins, caprins, lapins, chevaux, poissons. Paris, France: Institut National de la Recherche Agronomique; 2002

10. International guiding principles for biomedical research involving animals: The development of science-based guidelines for laboratory animal care NCBI Bookshelf. http://cioms.ch/publications/guidelines/1985_texts_of_ guidelines.htm.

11. Diario Oficial de la Federación: Especificaciones técnicas para la producción, cuidado y uso de los animales de laboratorio. Norma Oficial Mexicana NOM-062-ZOO-1999. México, D.F. Diario Oficial de la Federación, Miércoles 2 de Agosto 2001.

12. INRA: L'alimentation des animaux monogastriques: porc, lapin, volailles. Paris, France: Institut National de la Recherche Agronomique; 1984.

13. Adeola O: Digestion and balance technique in pigs. In Swine Nutrition. 2nd edition. Edited by Lewis AJ, Southern LL. Boca Raton, USA: CRC Press; 2001:903-916.

14. Le Bellego L, van Milgen J, Dubois S, Noblet J: Energy utilization of lowprotein diets in growing pigs. J Anim Sci 2001, 79:1259-1271.

15. de Souza RTC, Mar BB, Mariscal LG: Canulación de cerdos posdestete para pruebas de digestibilidad ileal: Desarrollo de una metodología. Téc Pecu Méx 2000, 38:143-150.

16. Fan MZ, Sauer WC, Hardin RT, Lien KA: Determination of apparent ileal amino acid digestibility in pigs: effect of dietary amino acid level. J Anim Sci 1994, 72:2851-2859.

17. AOAC: Official Methods of Analysis. 17th edition. Arlington, VA. USA: Assoc. Offic. Anal. Chem; 2000. 
18. Fenton TW, Fenton M: An improved procedure for determination of chromic oxide in feed and feces. Can J Anim Sci 1979, 59:631-634.

19. Henderson JH, Ricker RD, Bidlingmeyer BA, Woodward C: Rapid, accurate and reproducible HPLC analysis of amino acids. Amino acid analysis using Zorbax Eclipse AAA columns and the Agilent 1100 HPLC. Agilent Technologies 2000, Part No5980-1193E 2000:10. Agilent technologies home page at: www.agilent.com/chem/supplies.

20. Fan MZ, Sauer WC: Determination of apparent ileal amino acid digestibility in barley and canola meal for pigs with the direct, difference, and regression methods. J Anim Sci 1995, 73:2364-2374.

21. Furuya S, Kaji Y: Estimation of the true ileal digestibility of amino acids and nitrogen from their apparent values for growing pigs. Anim Feed Sci Technol 1989, 26:271-285.

22. Mariscal-Landín G, de Souza RTC: Endogenous ileal losses of nitrogen and amino acids in pigs and piglets fed graded levels of casein. Arch Anim Nutr 2006, 60:454-466.

23. Steel RGD, Torrie JH: Principles and procedures of statistics. A Biometrical approach. 2nd edition. New York: McGraw-Hill; 1980.

24. SAS version 9.2: Statistical Analysis Systems Institute User's guide. 92nd edition. : SAS Institute Inc; 2008

25. Moore SM, Stalder KJ, Beitz DC, Stahl CH, Fithian WA, Bregendahl K: The correlation of chemical and physical corn kernel traits with growth performance and carcass characteristics in pigs. J Anim Sci 2008, 86:592-601.

26. Wong JH, Marx DB, Wilson JD, Buchanan BB, Lemaux PG, Pedersen JF: Principal component analysis and biochemical characterization of protein and starch reveal primary targets for improving sorghum grain. Plant Sci 2010, 179:598-611.

27. Zhou Z, Wan HF, Li Y, Chen W, Qi ZL, Peng P, Peng J: The influence of the amylopectin/amylose ratio in samples of corn on the true metabolizable energy value for ducks. Anim Feed Sci Technol 2010, 157:99-103.

28. Gibbon BC, Wang $X$, Larkins BA: Altered starch structure is associated with endosperm modification in Quality Protein Maize. PNAS 2003, 100:15329-15334

29. Kil D, Kim B, Stein H: Feed energy evaluation for growing pigs. Asian-Aust J Anim Sci 2013, 26:1205-1217.

30. Szabó C, Halas V: Shortcomings of the energy evaluation systems in pigs: a review. Agric Conspec Sci 2013, 78:153-158.

31. Noblet J, van Milgen J: Energy value of pig feeds: effect of pig body weight and energy evaluation system. J Anim Sci 2004, 82(E-Suppl):E229-E238.

32. Cowieson AJ: Factors that affect the nutritional value of maize for broilers. Anim Feed Sci Technol 2005, 119:293-305.

33. Susenbeth A, Dickel T, Diekenhorst A, Höhler D: The effect of energy intake, genotype, and body weight on protein retention in pigs when dietary lysine is the first-limiting factor. J Anim Sci 1999, 77:2985-2989.

34. Burgoon KG, Hansen JA, Knabe DA, Bockolt AJ: Nutritional value of quality protein maize for starter and finisher swine. J Anim Sci 1992, 70:811-817.

35. Sullivan JS, Knabe DA, Bockholt AJ, Gregg EJ: Nutritional value of quality protein maize and food corn for starter and growth pigs. J Anim Sci 1989, 67:1285-1292.

36. Gunaratna NS, de Groote H, Nestel P, Pixley KV, McCabe GP: A metaanalysis of community-based studies on quality protein maize. Food Policy 2010, 35:202-210.

37. Azevedo RA, Arruda P: High-lysine maize: the key discoveries that have made it possible. Amino Acids 2010, 39:979-989.

38. Zarkadas CG, Yu JZ, Hamilton RI, Pattison PL, Rose NGW: Comparison between the protein quality of northern adapted cultivars of common maize and quality protein maize. J Agric Food Chem 1995, 43:84-93.

39. Stein $H H$, Sève B, Fuller MF, Moughan PJ, de Lange CFM: Invited review: amino acid bioavailability and digestibility in pig feed ingredients: terminology and application. J Anim Sci 2007, 85:172-180.

40. Stein HH, Fuller MF, Moughan PJ, Sève B, Moshentin R, Jansman AJM, Fernandez JA, de Lange CFM: Deffinition of apparent, true and standardized ileal digestibility of amino acids in pigs. Livest Sci 2007 109:282-285.

41. de Souza RTC, Aguilera BA, Mariscal-Landín G: Estimation of endogenous protein and amino acid ileal losses in weaned piglets by regression analysis using diets with graded levels of casein. J Anim Sci Biotechnol 2013, 4:36

42. Mariscal-Landín G, Sève B, Collèaux Y, LeBreton Y: Endogenous amino nitrogen collected from pigs with end to end ileorectal anastomosis is affected by the method of estimation and altered by dietary fiber. $J$ Nutr 1995, 125:136-146.

43. Yin Y-L, Li T-J, Huang R-L, Liu Z-Q, Kong XF, Chu W-Y, Tan B-E, Deng D, Kang P, Yin F-G: Evaluating standardized ileal digestibility in growing pigs. Anim Feed Sci Technol 2008, 140:385-401.

44. Pedersen C, Boisen S: Establishement of tabulated values for standardized ileal digestibility of crude protein and essential amino acids in common feedstuffs for pigs. Acta Agric Scand Sect A Anim Sci 2002, 52:121-140.

45. Urbaityte R, Mosenthin R, Eklund M, Piepho HP, Rademacher M: Determination of standardized ileal crude protein and amino acid digestibilities in protein supplements for piglets. Animal 2009, 3:1696-1705.

46. Yin $Y$, Gurung NK, Jeaurond $E$, Sharpe $P$, de Lange $C$ : Digestible energy and amino acid contents in Canadian varieties of sorghum, pearl millet, high-oil corn, high-oil-high-protein corn and regular corn samples for growing pigs. Can J Anim Sci 2002, 82:385-391.

47. Furuya S, Kaji Y: Additivity of the apparent and true digestible amino acid supply in barley, maize, wheat or soya bean based diets for growing pigs. Anim Feed Sci Technol 1991, 32:321-331.

doi:10.1186/2049-1891-5-26

Cite this article as: Mariscal-Landín et al:: Metabolizable energy, nitrogen balance, and ileal digestibility of amino acids in quality protein maize for pigs. Journal of Animal Science and Biotechnology 2014 5:26.

\section{Submit your next manuscript to BioMed Central and take full advantage of:}

- Convenient online submission

- Thorough peer review

- No space constraints or color figure charges

- Immediate publication on acceptance

- Inclusion in PubMed, CAS, Scopus and Google Scholar

- Research which is freely available for redistribution
C Biomed Central 